\title{
La différenciation sexuelle du cerveau par les gonadostéroïdes. Minirevue.
}

\author{
Ph. CIOFI \\ INSERM U.378 - Institut François Magendie, Bordeaux.
}

\section{RÉSUMÉ}

Cette revue expose les grands concepts relatifs à la différenciation sexuelle du cerveau des mammifères ainsi qu'ils furent formulés à partir d'expériences menées pour la plupart sur le rat de laboratoire. La différenciation sexuelle du cerveau est sous déterminisme génétique et épigénétique. Seul le déterminisme gonadostéroïdien épigénétique est abordé ici. La situation chez l'Homme sera évoquée en dernier. La différenciation sexuelle de l'anatomie et de la chimie du cerveau se conçoit avant tout comme nécessaire à l'émergence d'une différenciation fonctionnelle, les besoins de l'organisme suscités par la présence d'un ovaire ou d'un testicule étant différents. Au cours de l'organogenèse cérébrale, la gonade imposera donc son sexe lors de la construction de certains réseaux neuroniques qui constituent le "système voméronasal" dans le cerveau primitif. Celui-ci comprend en amont l'organe voméronasal sensible aux phéromones qui détermineront des adaptations comportementales et endocriniennes après traitement de l'information par l'hypothalamus. À bien des égards, le cerveau se sexualise comme se différencie l'appareil génital : le testicule détourne une structure bipotente d'un programme de différenciation féminine. La masculinisation du cerveau a lieu à un stade précoce de développement, pendant une période critique de sensibilité des neurones à l'action morphogénétique de la testostérone. L'empreinte testiculaire aura des effets d'organisation, permanents et irréversibles qui toucheront tous les niveaux d'action (métabolisme, chimie, pharmacologie...) et d'organisation (neurones et glie, axones, dendrites, synapses...) du tissu nerveux. D'une région cérébrale à l'autre, les mécanismes précis de sexualisation sont différents. Souvent, la testostérone agit après son aromatisation en ostradiol dans les neurones, mais le déterminisme stéroïdien peut aussi être purement androgénique ou mixte, andro-œstrogénique. Cette empreinte stéroïdienne régulera des processus d'apoptose, de croissance neuritique, d'acquisition de phénotype chimique. L'influence masculinisatrice du testicule étant la plus évidente, elle fut la mieux étudiée. Toutefois, la suppression expérimentale de l'empreinte testiculaire chez le mâle ne se traduit pas toujours par le développement inhérent des caractéristiques féminines. En effet, certains systèmes neuroniques nécessitent la présence de l'ovaire pour acquérir leurs caractéristiques définitives. Ainsi, le cerveau femelle n'est pas un cerveau indifférencié, et en cela s'écarte-t-il du schéma embryologique propre à l'appareil génital. Chez l'espèce humaine, des dimorphismes sexuels de l'anatomie et la chimie du cerveau sont aussi observables. Leurs conséquences fonctionnelles sont difficiles à circonscrire, autant que chez l'animal, d'ailleurs, et ce, essentiellement à cause de la complexité et de l'hétérogénéité locale du cerveau qui obscurcissent la lecture des rapports structure/fonction. Toutefois, des données histologiques concernant les cerveaux

Correspondance : Ph. Ciofi, INSERM U.378 - Institut François Magendie, 1 rue Camille Saint-Saëns, 33077 Bordeaux Cedex. philippe.ciofi@bordeaux.inserm.fr

Communication au XVIlème Congrès de la SALF, 7-8 décembre 2000, Bordeaux 
d'homosexuels et de transsexuels laissent à penser qu'orientation sexuelle et identité sexuelle auraient une base biologique. Chez l'Homme comme chez l'animal, le comportement sexuel - c'est-à-dire certains rapports sociaux - dépendrait, au moins en partie, de caractères innés.

Mots-clés : différenciation sexuelle du cerveau, dimorphismes sexuels, hypothalamus, cestradiol, testostérone, gonadolibérine.

Abréviations : A, androgènes ; AFP, alpha-fœetoprotéine ; APO, aire hypothalamique préoptique ; $A R$, récepteur nucléaire aux androgènes ; AROM, aromatase ; CCK, cholécystokinine ; DHT, dihydrotestostérone ; ER, récepteur nucléaire à l'œstradiol ; $\mathrm{E} 2$, œstradiol ; GnRH, gonadolibérine ; HSC, hyperandrogénie surrénalienne congénitale ; INAH3, noyau interstitiel de l'hypothalamus antérieur $\mathbf{n}^{\circ}$ 3 ; KO, knock out ; LH, hormone lutéinisante ; NLST, noyau du lit de la strie terminale ; NT, neurotensine ; SDN, noyau sexuellement dimorphe de l'aire préoptique ; $\mathrm{P}$, progestérone ; $\mathrm{PR}$, récepteur nucléaire à la progestérone ; $\mathrm{T}$, testostérone ; Tfm, testicule féminisant.

\section{INTRODUCTION}

Linvention de la sexualité par le vivant a conduit à une explosion de la biodiversité. La fonction de reproduction, qui a la particularité d'être à la fois le moteur et le substrat de l'évolution, s'est diversifiée dans son expression avec la création d'espèces nouvelles : celles-ci sont en compétition pour l'occupation des biotopes et dans la nécessité de maintenir un cloisonnement génique. D'où l'émergence d'un système nerveux central capable d'un contrôle relativement autonome de la gamétogenèse assorti d'un comportement reproducteur approprié. Ainsi, en 450 millions d'années, les Vertébrés se sont-ils dotés du "système voméronasal" [13], une composante du cerveau primitif qui reçoit en amont des informations phéromonales et extéroceptives dont le traitement induit une adaptation endocrinienne et motrice (Figure 1).

Par exemple, chez les Mammifères, puisque les sexes sont séparés, le système voméronasal est responsable, à la fois, de la coordination de l'endocrinologie et du comportement sexuels de l'individu, et de la coïncidence spatio-temporelle de cette coordination entre les différents

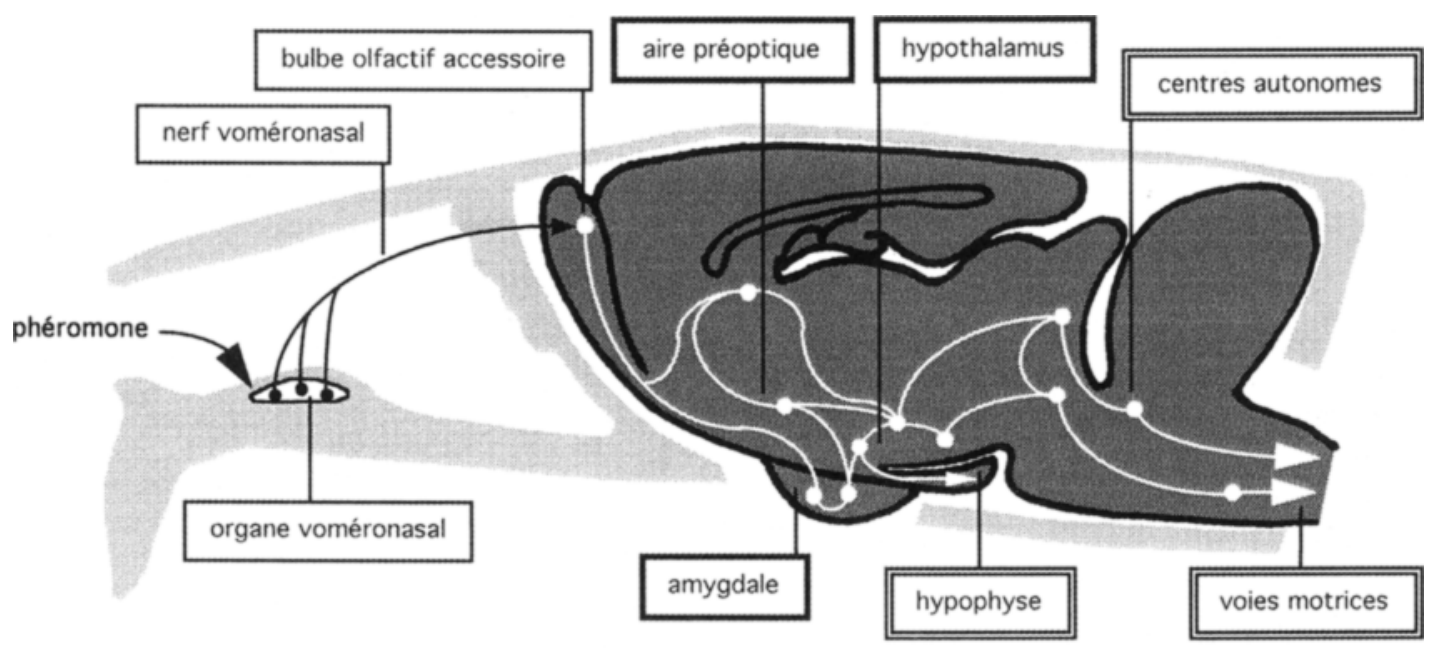

information phéromonale $\quad$ intégration limbique

systèmes effecteurs

Figure 1 : Organisation du système voméronasal. Les circuits indiqués sont sensibles aux gonadostéroüdes 
individus : des millions de neurones sont dédiés à cette tâche dans le cerveau humain. Les réseaux neuroniques centraux gérant la production et la réunion des gamètes sont donc complexes, et ils demeurent encore mal compris. Il apparaît aujourd'hui que certains d'entre eux sont construits différemment selon la nature de la gonade. Cela n'a rien d'étonnant, les besoins de l'organisme suscités par la présence d'un ovaire ou d'un testicule étant différents. Pourtant, la notion de la différenciation sexuelle du cerveau n'est pas intuitive, elle ne s'est imposée que progressivement, au cours des trente dernières années, suite à l'accumulation de résultats intrigants provenant d'études physiologiques, comportementales et histologiques, suite aussi à la découverte de la présence de tous les récepteurs nucléaires aux gonadostéroïdes dans de nombreuses régions du système nerveux central.

La petite revue qui suit rappelle les concepts fondamentaux de la discipline tels qu'ils ont été établis chez l'animal par des expériences désormais classiques. Certains concepts, comme nous le verrons, sont directement applicables à l'Homme, alors que d'autres n'ont pas encore trouvé leur place en physiologie humaine. Tous ont une portée heuristique, voire humaniste, indéniable. Des références bibliographiques ne seront pas données systématiquement, mais un échantillonnage d'articles est proposé pour chaque sujet abordé.

\section{DIFFÉRENCIATION SEXUELLE FONCTIONNELLE : AXE HYPOTHA- LAMO-HYPOPHYSO-GONADIQUE}

$$
[15,21,33]
$$

\section{Premières traces : aberRation naturelle.}

Les éleveurs savaient depuis longtemps que l'orchidectomie atténue l'agressivité des mâles. Des expériences de castration-réimplantation de testicule chez le coq avaient aussi confirmé le rôle inducteur de la glande vers la maturité sexuelle. Toutefois, les éleveurs avaient aussi remarqué que dans un troupeau, certaines vaches peuvent avoir un comportement spontané de monte vis-à-vis de leurs congénères. Ces femelles à comportement masculin (les freemartins) sont toujours issues d'une gros- sesse gémellaire avec un fotus mâle et demeurent stériles car leurs organes génitaux sont atrophiés. La première explication mécaniste de cette observation séculaire est apportée par Keller et Tandler (1916) et par Lillie (1917) qui constatent que des anastomoses vasculaires unissent les deux chorions dans l'utérus, et émettent l'hypothèse qu'une substance issue du testicule du jumeau mâle influence le développement du jumeau femelle.

Les travaux de Frank Lillie vont stimuler la recherche sur la détermination du sexe chez l'embryon, et, pendant la première moitié du vingtième siècle, embryologie, endocrinologie, neuroendocrinologie et neurophysiologie de la reproduction évolueront de concert, les unes s'enrichissant des progrès des autres [14, 20]. Le résultat principal de cette évolution des connaissances fut la constitution d'une théorie hormonale et génétique de la différenciation de l'appareil génital des Mammifères : le chromosome $\mathrm{Y}$ décide de la constitution d'un testicule qui à son tour impose son sexe à une structure bipotente dont le programme inhérent de développement est féminin. Cette théorie sera mise à l'épreuve par les neurobiologistes étudiant le cerveau reproducteur.

\section{DES RATTES STÉRILES : ACTION IRRÉVERSIBLE DU TESTICULE SUR L'HYPOTHALAMUS.}

La gestation chez le Rat est de 21 jours. Les nouveau-nés sont encore peu développés à la naissance, mais l'appareil génital interne est achevé. La puberté survient 36 jours post-partum (première ovulation) chez la femelle, 50 jours (premiers gamètes mobiles) chez le mâle. Les corps jaunes sont visibles à l'examen macroscopique. Ces caractéristiques font de ce rongeur un sujet expérimental de choix. De plus, le déterminisme de l'ovulation est très rigide chez les Rongeurs : la rétroaction positive de l'œstradiol (E2) sur la sécrétion de lutéotropine (LH) est un réflexe neuroendocrinien qui ne s'observe que chez la femelle. Si ce modèle animal s'écarte à cet égard de la physiologie des Primates, cette situation s'est avérée avantageuse pour l'expérimentation.

Répétant chez le Rat à l'aide d'expériences et de contre-expériences la situation particulière aux freemartins, plusieurs laboratoires font les 
observations suivantes concernant le déterminisme de l'ovulation. Les femelles qui sont ovariectomisées à la naissance et qui reçoivent une greffe d'ovaire à l'âge adulte sont capables de faire des corps jaunes. Les femelles qui, à la naissance, reçoivent une greffe testiculaire ou sont traitées à la testostérone (T) sont stériles à l'âge adulte, présentent des ovaires sans corps jaunes, n'ont pas de rétroaction positive à l'E2. Les mâles orchidectomisés à l'âge adulte et recevant une greffe d'ovaire sont incapables de faire des corps jaunes. Les mâles orchidectomisés à la naissance peuvent, à l'âge adulte, répondre positivement à l'E2 et promouvoir la formation de corps jaunes dans les greffes ovariennes.

Ces expériences ont fait apparaître que le milieu interne féminin est capable de soutenir de façon inhérente le fonctionnement de l'ovaire, alors que cette faculté est irréversiblement perdue chez le mâle suite à l'action masculinisatrice de la $\mathrm{T}$ à un stade précoce de développement. Chez le rat mâle comme chez l'homme, une crise testiculaire s'observe en période pré- et néonatale.

Alors que ces travaux sont réalisés, la théorie neurovasculaire du contrôle hypothalamique de l'hypophyse se répand dans la communauté des physiologistes. Conséquence directe de cette évolution des connaissances, le site d'action masculinisatrice de la $\mathrm{T}$ est recherché. Les expériences les plus difficiles mais remarquables consistent en une série de greffes croisées d'ovaire ou d'hypophyse entre femelles stérilisées néonatalement par la T (donneuses) et femelles normales (receveuses). L'ovaire d'une femelle androgénisée à la naissance, une fois replacé dans un contexte physiologique normal est capable de former des corps jaunes. De même, l'antéhypophyse d'une femelle androgénisée est capable d'induire la formation de corps jaunes dans les ovaires d'une femelle normale.

Ni l'ovaire, ni l'hypophyse ne sont donc la cible de l'action masculinisatrice de la T. C'est un étage plus haut, dans l'hypothalamus, que le testicule laisse son empreinte.

La découverte du rôle essentiel de l'hypothalamus dans le contrôle de l'homéostasie a rapi- dement suscité, avec l'avancée des techniques de microchirurgie et de stimulation électrique, des explorations systématiques des rapports structure-fonction dans le cerveau. En particulier, avant la découverte des facteurs hypothalamiques hypophysiotropes et la mise au point de l'immunocytochimie, il apparut clairement que la région préoptique, située en avant et à la base du diencéphale, est le siège de la commande centrale de l'ovulation chez la plupart des espèces d'infra-primates. Ainsi, sa stimulation électrique déclenche la rupture folliculaire chez une femelle normale alors qu'elle demeure inefficace chez une femelle androgénisée à la naissance. La $\mathrm{T}$ agit donc pour modifier irréversiblement le fonctionnement de réseaux neuroniques précis.

\section{DES RATTES PAS TOUJOURS STÉRILES : PÉRIO- DE CRITIQUE D'ACTION DE LA TESTOSTÉRONE.}

Les premières expériences d'androgénisation donnèrent des résultats variables : certaines femelles semblaient insensibles au traitement. Ainsi, une injection unique de $T$ est efficace dans tous les cas si elle est réalisée au jour post-natal 5, dans quelques cas au jour postnatal 10, jamais au jour post-natal 20. Par ailleurs, une femelle androgénisée en fin de gestation donnera naissance à des femelles infertiles.

En conclusion, l'hypothalamus ne peut être masculinisé durant son développement que pendant une période critique de sensibilité à la T.

\section{DIFFÉRENCIATION SEXUELLE FONCTIONNELLE : COMPORTE- MENT SEXUEL}

\section{Le COMPortement SEXUEL du Cobaye : DUA- LITÉ DE L'EMPREINTE STÉROÏDIENNE.}

Dans un article classique de 1959, le groupe de Phoenix démontre la dualité d'action des stéroïdes sexuels. La longue période de gestation du Cobaye (65 jours) aboutit à la naissance de petits déjà bien développés. Les expérimentateurs ont comparé l'effet d'un traitement prolongé au propionate de $\mathrm{T}$, administré soit pendant la gestation soit après la naissance, sur l'expression ultérieure à l'âge adulte, du comportement sexuel des femelles (alors ovariecto- 
misées et traitées à l'E2 et la progestérone $(\mathrm{P})$ pour permettre le réflexe). Ils font les observations suivantes. Le traitement prénatal prolongé à la $\mathrm{T}$ a aboli ou réduit considérablement la capacité d'expression du réflexe de lordose, même si la T est appliquée à des doses insuffisantes pour masculiniser l'appareil génital externe. L'expression du comportement de monte est accrue. En revanche, même aux doses les plus fortes, le traitement prolongé postnatal à la $\mathrm{T}$ n'a pas eu d'effet durable. Chez les mâles, le traitement pré- ou postnatal n'a pas d'effet. En somme, la présence de T pendant le développement des femelles semble avoir eu un effet irréversible de déprogrammation ou reprogrammation du sexe comportemental par rapport au sexe génétique.

Suite aux travaux de Phoenix, une dichotomie conceptuelle guidera la recherche sur la différenciation sexuelle du cerveau. Les effets des gonadostéroïdes qui sont observables chez les individus castrés à l'âge adulte sont appelés "effets d'activation" : ils sont temporaires et réversibles (régulation des circuits reproducteurs). Ils sont naturellement observés à partir de la puberté. Les effets des gonadostéroïdes qui sont expérimentalement dissociables du sexe génétique sont appelés "effets d'organisation" : ils sont permanents et irréversibles (constitution des circuits). Ils sont naturellement observés durant l'organogenèse cérébrale (Figure 2).

L'expression du comportement étant le reflet $\mathrm{du}$ fonctionnement cérébral, la différenciation sexuelle du cerveau semble donc s'installer selon le principe gouvernant la constitution de l'appareil génital : un cerveau intrinsèquement femelle et bipotent, masculinisé par l'empreinte testiculaire.

SEXE GÉNÉTIQUE

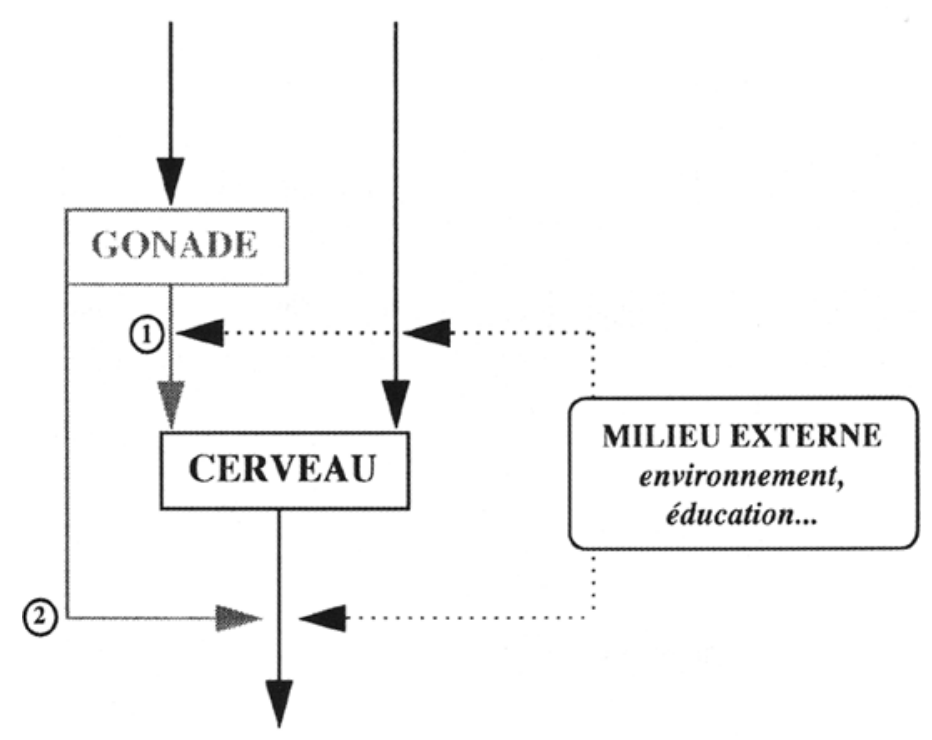

SEXE PHÉNOTYPIQUE

endocrinologie \& comportement

Place de la gonade dans la différenciation sexuelle du cerveau

(1) Organisation des réseaux, période périnatale

(2) Activation des réseaux, puberté

Figure 2 


\section{IV. ÉTUDES DES DIMORPHISME SEXUELS DANS L'HYPOTHALAMUS}

$[2,6,8,9,17,29]$

\section{DE LA FONCTION À LA STRUCTURE : L'AIRE HYPOTHALAMIQUE PRÉOPTIQUE DU RAT.}

L'aire préoptique (APO) est impliquée dans le contrôle de l'ovulation et le comportement sexuel. Ces données poussèrent Raisman et Field, deux neurocytologistes spécialistes en microscopie électronique, à comparer l'ultrastructure de l'APO entre les sexes. Ils découvrirent une différence très nette dans le nombre d'un certain type de synapses axo-dendritiques dans une région dorsale. Leurs travaux publiés en 1971 et 1973 eurent un retentissement énorme en ce qu'ils établirent pour la première fois une différence d'organisation du cerveau entre les sexes. Mais ces travaux laissèrent à penser que seules de fines différences d'ultrastructure seraient observées. Ce n'est qu'en 1978 que, dans la région même étudiée par Raisman et Field, le groupe de Gorski démontre l'existence d'un dimorphisme sexuel spectaculaire de la macrostructure de l'APO : le noyau central comporte environ cinq fois plus de neurones chez les mâles que chez les femelles (Figure 3). Ce modèle, le noyau central ou SDN (sexually dimorphic nucleus), est exceptionnel en ce qu'il ne nécessite qu'une coloration histologique classique pour être révélé et qu'il peut être observé à l'aide du microscope photonique le plus simple.

Par la suite, de nombreux travaux morphomé-
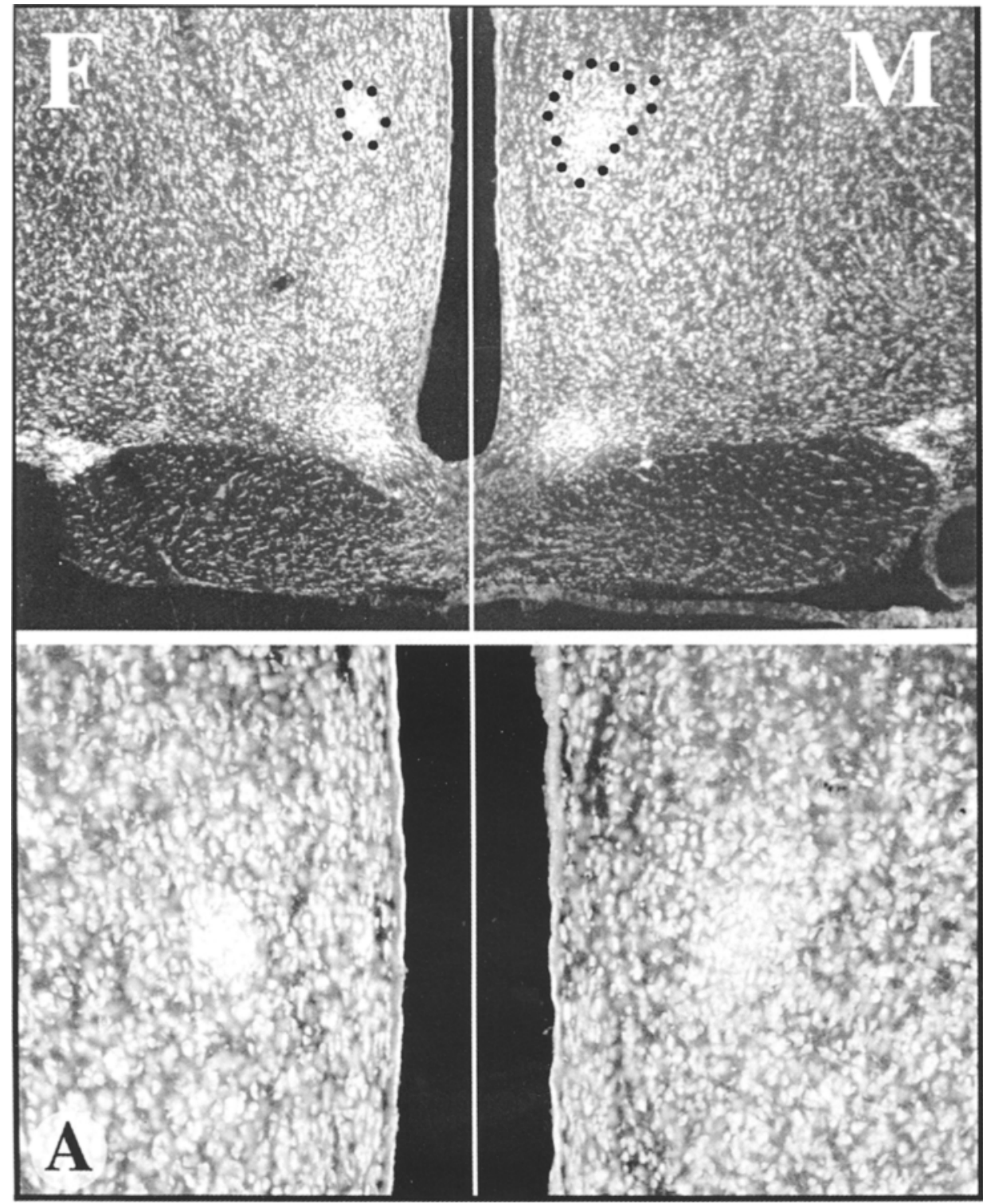

Figure 3 : Exemple de dimorphisme sexuel de la structure du cerveau : coloration histologique fluorescente nucléaire par le bromure d'éthydium de coupes frontales dans l'aire hypothalamique préoptique du rat et révélant par confrontation la différence de développement du noyau sexuellement dimorphe (SDN, pointillés en haut) entre femelle (F) et mâle (M). En bas, vues du SDN à plus fort grossissement. 
triques réalisés sur le SDN ont testé l'hypothèse que les effets d'activation et d'organisation des stéroïdes sexuels sur l'endocrinologie et le comportement sexuels (fonction) sont aussi observables concernant le tissu nerveux (structure). Ces travaux sont présentés ci-dessous.

\section{Rôle DE LA TESTOSTÉrone.}

Les différentes expériences de gonadectomie associée ou non à des traitements gonadostéroïdiens substitutifs furent répétées, et le nombre de neurones du SDN compté dans chaque individu. La gonadectomie à l'âge adulte ne modifie pas le dimorphisme sexuel dans le nombre de neurones du SDN.

L'orchidectomie à la naissance abaisse le nombre de neurones du SDN à un niveau qui est celui des femelles normales. Cet effet est empêché par un traitement substitutif à la $\mathrm{T}$ administré néonatalement aux femelles, ce traitement augmente le nombre de neurones à un niveau qui atteint celui des mâles normaux.

Ainsi, la T est capable de renverser le sexe phénotypique du SDN. Elle exerce une fonction morphogénétique (assurant la constitution du tissu nerveux) et a un effet épigénétique (s'exerçant indépendamment du sexe génétique de l'individu).

Pourtant, mâles et femelles naissent avec un nombre identique de neurones dans le SDN. À partir du quatrième jour de vie extra-utérine, des neurones commencent à mourir dans le SDN des femelles. Chez les nouveau-nés mâles normaux, ou chez les femelles traitées à la $\mathrm{T}$ pendant cette période, les neurones survivent.

La $\mathrm{T}$ agit donc pour enrayer un programme d'apoptose neuronale intrinsèquement programmé dans le SDN des deux sexes (le SDN ressemblerait à une structure génitale wolffienne).

\section{MODE D'ACTION de LA TEstostérone.}

Un effet paradoxal de l'E2 est sa capacité à stériliser les femelles après traitement néonatal à fortes doses. Quand le SDN de ces individus est analysé, on y retrouve un nombre masculin de neurones. L'hormone femelle masculinise l'organisation du SDN aussi bien que l'hormone mâle. En fait, un traitement néonatal des mâles par un antagoniste du récepteur aux androgènes (AR, androgen receptor) ne démasculinise pas le SDN. De plus, les mâles porteurs d'une mutation inactivatrice de l'AR (testicule féminisant) présentent un SDN masculinisé : l'AR ne semble pas avoir de rôle déterminant dans la différenciation sexuelle du SDN. Enfin, les mâles ayant reçu un traitement périnatal par un antagoniste des récepteurs à l'E2 (ER, estrogen receptor) présentent un SDN démasculinisé et une injection néonatale intra-APO d'un oligonucléotide anti-sens bloquant l'ARNm de ERo, protège le SDN des femelles de la masculinisation par la $\mathrm{T}$.

En conclusion, l'effet organisateur irréversible du testicule sur la structure du SDN semble être médié par l'ER.

L'observation, d'une part que le dérivé non-aromatisable de la T, la dihydrotestostérone (DHT), ne masculinise pas le SDN de femelles néonatales, et d'autre part que les inhibiteurs de l'aromatase (AROM) démasculinisent le SDN des mâles, indique que la différenciation sexuelle du SDN est due à l'aromatisation de la T en E2.

Puisque les neurones du SDN possèdent à la fois les ER et l'AROM, il est admis que l'aromatisation de la $\mathrm{T}$ se fait localement, dans les cellules cibles elles-mêmes.

\section{DE L'INDIFFÉRENCLATION FÉMININE}

En l'absence de gonade, c'est le sexe femelle qui s'impose lors du développement de l'appareil génital : le modèle indifférencié serait intrinsèquement femelle. Cette hypothèse a été testée sur le SDN par le traitement néonatal de femelles par un antagoniste des ER (tamoxifène) ou par l'injection intra-APO d'un oligonucléotide antisens bloquant l'ARNm de $\mathrm{ER} \alpha$. Chez les femelles traitées, le SDN contient moins de neurones que chez les femelles normales. Il semble donc que le développement du SDN dans le sexe femelle requiert aussi une influence de l'E2 : le SDN ne reste pas indifférencié chez les femelles.

C'est l'intensité de l'empreinte stéroïdienne qui permettrait l'émergence de dimorphismes sexuels dans certaines régions du cerveau. La persistance d'une organisation indifférenciée reposerait sur l'absence totale d'imprégnation stéroïdienne. 


\section{UNE PRoteCtION FÉMININE}

Le fotus femelle, ainsi que la mère, produisent de l'E2. Puisque 1'E2 agissant principalement via $\mathrm{ER} \alpha$ enraye l'apoptose des neurones du SDN chez les mâles, ce mécanisme devrait aussi complètement masculiniser les femelles. Toutefois, dans les fotus des deux sexes, il circule en très fortes concentrations une protéine hépatique que les neurones produisent aussi et qui lie l'E2 avec une forte affinité : l' $\alpha$-foetoprotéine (AFP). Chez les femelles, l'E2 circulant est donc piégé par l'AFP. Chez les mâles, la T qui ne se lie pas à l'AFP est donc libre de pénétrer le SDN. Il est donc supposé que dans les neurones, l'AFP jouerait le rôle d'un tampon de la concentration intracellulaire en E2, par des mécanismes qui demeurent inconnus. La petite dose d'E2 nécessaire à la différenciation du SDN des femelles serait libérée par le complexe AFP-E2 intracytoplasmique.
La $\mathrm{T}$ aromatisée en E2 dans les neurones du SDN des mâles serait concentrée par ceux-ci au-delà des facultés de liaison de l'AFP, résultant en une imprégnation suffisante à déclencher la cascade moléculaire d'inhibition de l'apoptose (Figure 4).

\section{Précisions sur la période critique}

Comme nous l'avons vu plus haut, la masculinisation des rattes par les androgènes peut être obtenue in utero comme en période néonatale. Une série d'expériences a révélé les bornes développementales de la période critique de sensibilité de l'hypothalamus aux effets d'organisation de la T. Chez le Rat, le SDN des femelles peut être masculinisé depuis le 18ème jour de la gestation jusqu'au 4ème jour de vie extra-utérine. Cette période critique possède donc des bornes abruptes. Le 4ème jour postnatal marque le début de la phase d'apoptose neuronale dans le

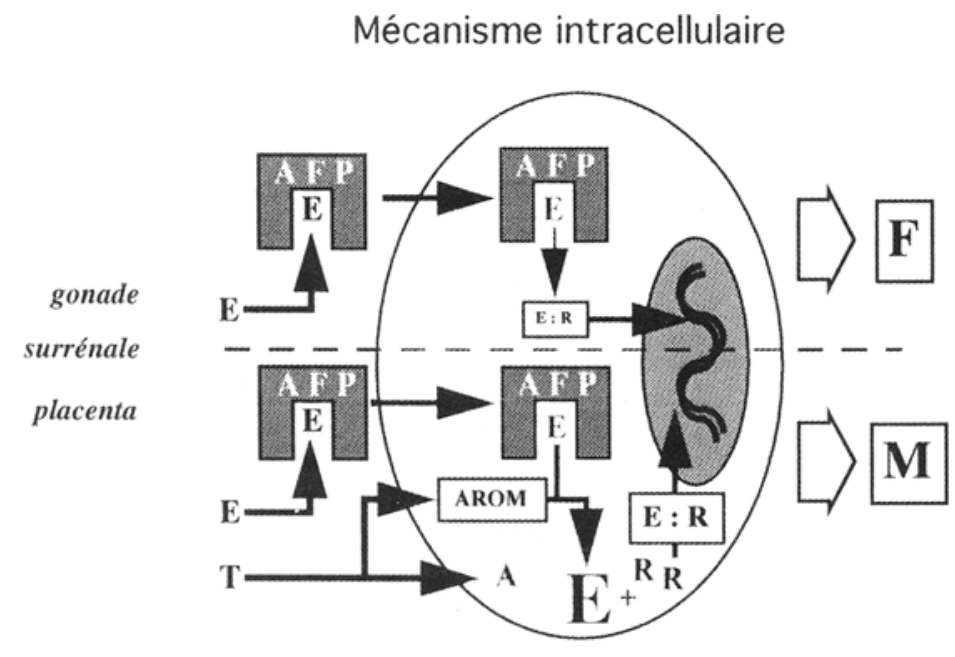

A, androgènes; AROM : aromatase; E : østradiol;

$\mathbf{R}$ : récepteur; $\mathrm{T}$ : testostérone; $\mathrm{AFP}$ : alpha-fétoprotéine

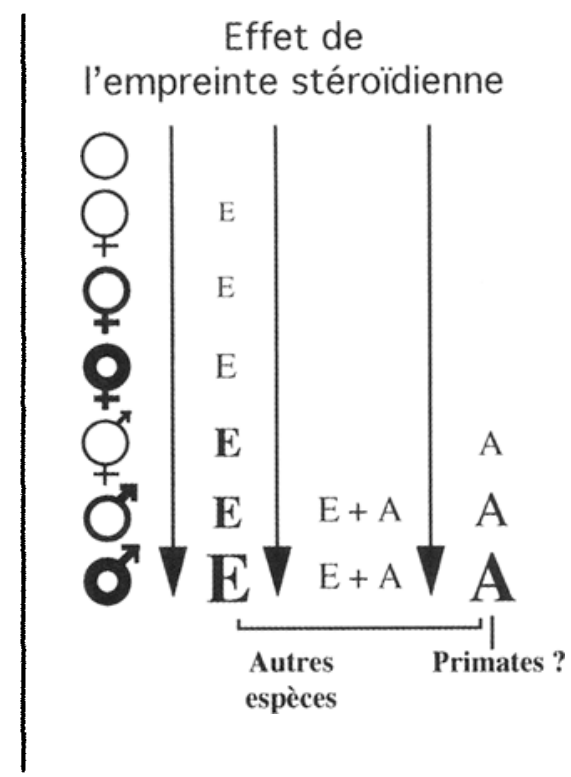

Mécanismes prouvés :

Chez les non-primates: Le testicule embryonnaire libère de la T qui est aromatisée en E par le cerveau. Chez les femelles, l'E circulant est piégé par l'AFP d'origine hépatique et cérébrale. Dans les deux sexes, l'AFP agirait comme un tampon de l'E intracellulaire. Certaines régions cérébrales ne sont sensibles qu'à l'E, d'autres nécessitent la coopération de l'E et des A, d'autres encore dépendent des A seuls. La progestérone semble aussi impliquée.

Hypothèse :

Chez les primates : l'AFP est remplacée par une autre protéine transporteur (?). Les A sont seuls responsables de la différenciation sexuelle (?).

Figure 4 : Déterminisme et mécanisme de la différenciation sexuelle du cerveau 
SDN : quand l'apoptose est déclenchée, les neurones sont devenus insensibles à l'effet protecteur de la T. Le 18ème jour de la gestation, date d'entrée du SDN dans la période de sensibilité à la T, est caractérisé chez l'embryon mâle par une élévation forte et soudaine de la sécrétion testiculaire de T et de l'activité de l'AROM hypothalamique.

Ainsi, différents évènements périphériques et centraux coïncident dans l'embryon mâle afin d'assurer la différenciation sexuelle du cerveau. De plus, des travaux récents ont montré l'expression périnatale du récepteur à la $\mathrm{P}$ (le PR) dans le SDN des mâles exclusivement, suggérant une participation de la mère au processus.

\section{ACTIONS POSSIBLES DE L'ESTRADIOL LORS DE LA DIFFÉRENCIATION SEXUELLE}

L'absence d'effet "macroscopique" des antagonistes des AR sur la masculinisation du SDN n'exclut pas, bien sûr, que les $A R$ puissent intervenir dans l'organisation fine de l'aire préoptique. Ce champ d'investigation reste ouvert. Linfluence profonde des ER a, quant à elle, suscité plus d'intérêt. En particulier, des études in vitro ont comparé le devenir d'explants d'APO fœtale maintenus en survie pendant quatre jours dans un milieu nourricier supplémenté ou non en E2. Les explants cultivés en présence d'E2 montrent une importante croissance de leurs extensions axoniques et dendritiques, à la fois en termes d'élongation et de ramification : l'E2 agit donc comme un facteur de croissance. Des travaux récents ont insisté sur ce rôle trophique de l'E2 dont les voies de signalisation intracellulaire sont en partie communes avec celles des neurotrophines.

La période critique de sensibilité de l'hypothalamus à l'E2 est aussi caractérisée par des mouvements morphogénétiques, des migrations neuronales et des acquisitions de phénotype chimique, et l'on doit s'attendre à une participation différentielle de l'E2 dans tous ces phénomènes. Une récente étude in vitro sur des cellules transfectées de neuroblastome a démontré l'action différentielle de $\operatorname{ER} \alpha$ et $\operatorname{ER} \beta$ sur divers paramètres de la croissance neuritique.

\section{Différenciation SeXuelle du PHÉnOtyPe CHIMIQUE DES NEURONES}

La découverte d'un nombre toujours croissant de neuropeptides, ces messagers à rôle neuromodulateur, a permis aux immunocytologistes de dresser une cartographie de la neuroanatomie chimique du cerveau. Certains circuits peptidergiques se distinguent de l'ensemble par leur réactivité à la gonadectomie. Leur contenu en neuropeptide immunoréactif, ou leur intensité d'expression d'ARNm visualisée par les techniques d'hybridation in situ, sont altérés réversiblement par la castration et le traitement stéroïdien substitutif, ce qui signale leur implication dans les mécanismes de contrôle de la reproduction. Lors de la différenciation sexuelle du cerveau, l'empreinte testiculaire déterminera irréversiblement le répertoire chimique des neurones, en qualité et en intensité. Par exemple, dans un circuit probablement impliqué dans le comportement parental, qui relie entre elles deux régions limbiques, le noyau du lit de la strie terminale (NLST) et le septum latéral, le neuropeptide vasopressine (ou hormone anti-diurétique) est produit en faible quantité chez la femelle par rapport au mâle. Dans un autre système de neurones, le circuit neuroendocrinien à gonadobérine $(\mathrm{GnRH})$, les neuropeptides cholécystokinine (CCK) et neurotensine (NT) ne sont exprimés que chez la femelle (figure 5).

Ainsi en est-il de dizaines de populations neuroniques du cerveau, différentes dans leur structure et dans leur phénotype chimique et pharmacologique, ce qui rend compte des différences de fonction. À l'évidence, la découverte de la différenciation sexuelle du cerveau rend cet organe encore plus complexe qu'on pouvait l'imaginer.

\section{PAS DE RÈGLE GÉNÉRALE POUR LA SEXUALISATION DU CERVEAU}

\section{RAPPELS DES GRANDS CONCEPTS ISSUS DE} L'ÉTUDE DE L'HYPOTHALAMUS DU RAT

Durant une période critique de sensibilité du tissu nerveux à la $\mathrm{T}$, la suractivité transitoire du testicule embryonnaire impose un programme de développement masculin à des structures bipotentes qui quitteront une voie d'or- 

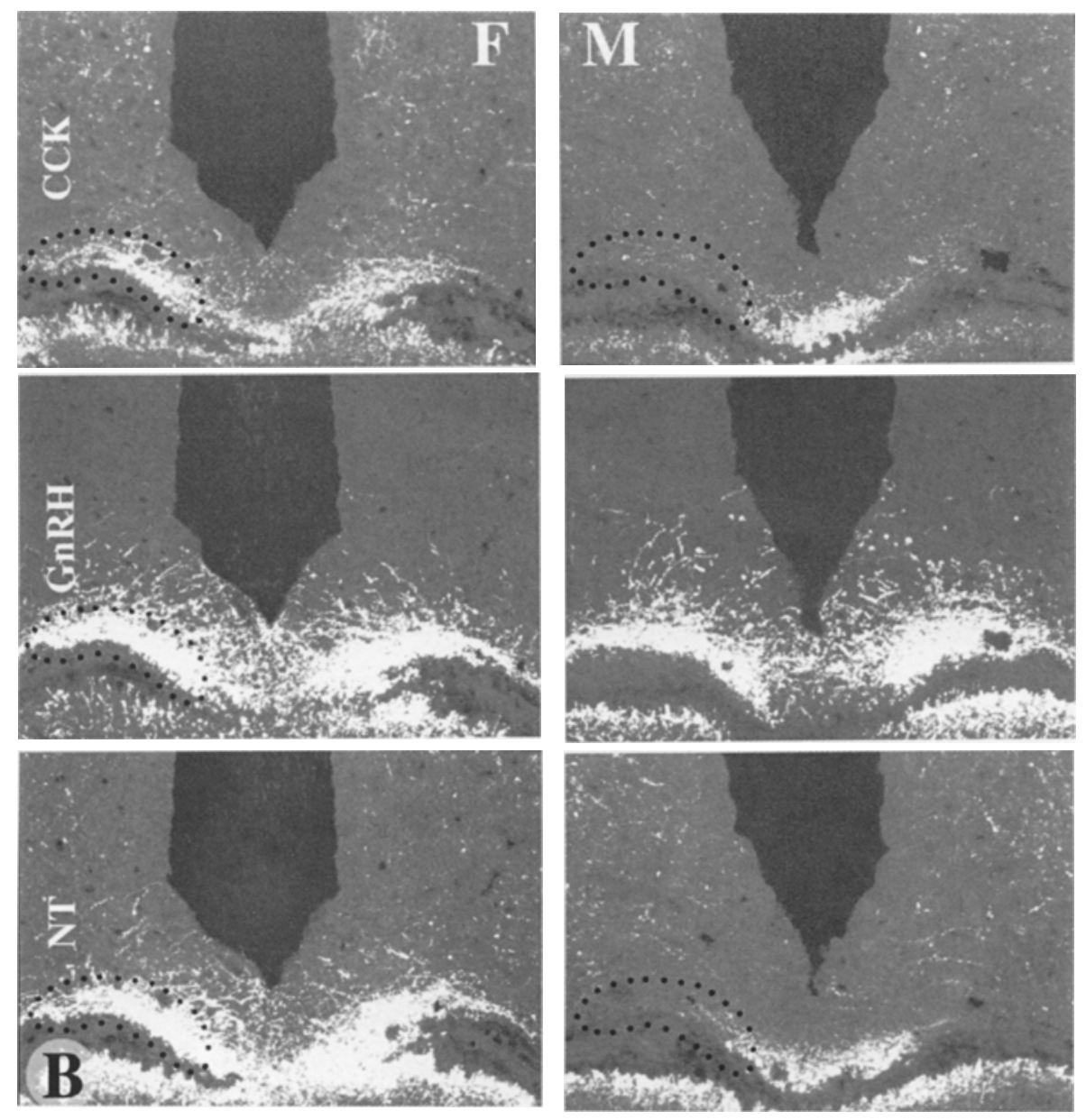

Figure 5 : Exemple de dimorphisme sexuel de la chimie du cerveau : comparaison sur trois coupes frontales dans l'hypothalamus médiobasal du rat de la distribution du marquage en immunofluorescence pour trois neuropeptides, la cholécystokinine (CCK, en haut), la gonadolibérine (GnRH, au centre) et la neurotensine (NT, en bas). Les fibres neurosécrétrices à GnRH ne contiennent la CCK et la NT que chez la femelle (F), comme l'indiquent les plages délimitées par les pointillés et qui sont vides de marquage chez le mâle (M). (Clichés de l'auteur ; Pr. G. Tramu, Univ. Bordeaux I, pour les immunsérums).

ganisation femelle suite à l'action irréversible de la $\mathrm{T}$ aromatisée en $\mathrm{E} 2$. L'AFP protège le cerveau femelle de l'action différenciatrice de l'E2.

\section{LA DIFFÉRENCIATION SEXUELLE DU CERVEAU: UNE SUITE DE CAS PARTICULIERS}

L'APO de l'hypothalamus du Rat s'est révélée un modèle expérimental extrêmement fructueux. La découverte des mécanismes fondamentaux de sa sexualisation ont permis l'analyse comparée d'autres régions centrales et ce, dans plusieurs espèces. Il apparut rapidement que chaque région du système nerveux central obéit à des règles ou à un assemblage de règles qui lui sont propres. De plus, certains dimorphismes sexuels se retrouvent dans une espèce mais pas dans une autre, ce qui reflète certainement l'adaptation de l'espèce à son environ- nement, sa stratégie de reproduction, ses tendances évolutives propres.

Le rôle important des ER, et l'absence d'influence marquante des AR dans la sexualisation du SDN n'est pas une règle générale. Par exemple, les motoneurones lombaires contrôlant la musculature du pénis dépendent d'une activation des $\mathrm{AR}$ et de l'influence trophique de la musculature qu'ils innervent pour leur survie en période néonatale. Ils sont absents chez les femelles ainsi que chez les mâles porteurs d'un testicule féminisant, et suite à l'activation des AR, ces neurones dépendent aussi d'une coopération AR-ER. Ce groupe de motoneurones existe dans notre espèce (noyau d'Onuf) et y survivent en petit nombre chez la femme.

Les gonadostéroïdes peuvent agir, indépen- 
damment de leurs récepteurs nucléaires, directement sur la membrane des neurones en se liant soit à des récepteurs propres (que l'on recherche avec grand intérêt), soit à des récepteurs de neurotransmetteurs. Bien que difficiles à prouver expérimentalement, les effets non-génomiques, membranaires, des gonadostéroïdes dans la sexualisation du cerveau ne sauraient être écartés.

La fonction protectrice de l'AFP durant la différenciation du cerveau est documentée chez le Rat, mais peu d'éléments sont disponibles concernant les Primates.

La constitution d'un cerveau mâle ne nécessite pas toujours la survie de neurones surnuméraires. Dans certaines régions du cerveau, comme dans la région diencéphalique de l'aire antéroventrale périventriculaire, c'est la présence de $\mathrm{T}$ aromatisée en $\mathrm{E} 2$ qui déclenche l'apoptose chez les mâles, conduisant à un avantage numérique chez les femelles dans cette région.

Le cerveau femelle n'est pas indifférencié. Il apparaît en effet que certains dimorphismes sexuels ne peuvent émerger en l'absence de l'ovaire.

Les stéroïdes ne sont pas les seuls facteurs de différenciation sexuelle. Les neurotransmetteurs libérés dans le cerveau embryonnaire à l'entrée en activité des circuits en construction ont probablement aussi une influence profonde. Cet aspect pharmacologique de la différenciation du cerveau est à prendre en compte en médecines vétérinaire et humaine.

La différenciation sexuelle du cerveau ne se déclenche pas de façon purement épigénétique. De nombreuses expériences ont obtenu des résultats inexplicables dans un cadre excluant une composante génétique.

\section{LA NOTION D'EFFETS D'ACTIVATION ET D'OR- GANISATION}

Les notions de permanence (organisation) et de réversibilité (activation) des effets des gonadostéroïdes sont relatives en ce qu'elles dépendent de la mesure. Le plus souvent, la permanence d'un effet n'est examinée qu'à moyen terme. Tel effet d'un traitement néonatal est jugé permanent s'il peut être [encore] observé chez le jeune adulte, c'est-à-dire à trois ou quatre mois d'âge chez le rat de laboratoire. La réversibilité se comprend comme la restitution globale, statistique, d'un paramètre parmi tant d'autres caractérisant un système neuronique. Néanmoins, cette dichotomie s'applique parfaitement à des processus comme la mort cellulaire et l'acquisition d'un répertoire pharmacologique, phénomènes irréversibles, ainsi qu'à des phénomènes réversibles comme les fluctuations des taux d'expression d'ARN messagers ou de couverture synaptique neuronale que l'on observe au cours du cycle ovarien dans certaines régions limbiques en réponse aux rétroactions gonadostéroïdiennes.

Les effets d'organisation et d'activation des gonaḍostéroïdes se matérialisent à tous les niveaux d'action (activité électrique, métabolisme, panoplie pharmacologique, synthèse des messagers chimiques...) et d'organisation (neurones et cellules gliales, quantités et dimensions des cellules, arborisation des axones et dendrites, quantité et nature des synapses...) du tissu nerveux, et dans un grand nombre de régions cérébrales. Dans son acceptation la plus large, la dichotomie organisation/activation évoque la succession de deux étapes dans le développement cérébral :

a) constitution puis mise au repos des circuits neuroniques à compétence reproductive pendant l'organogenèse, suivie de

b) leur ré-activation à la puberté.

\section{LA NOTION DE PÉRIODE CRITIQUE}

Celle-ci est à son tour liée aux notions de permanence et de réversibilité. La période critique peut se comprendre comme une fenêtre temporelle de sensibilité du neurone à un signal. Elle peut aussi être définie comme la partie du développement au cours de laquelle des événements irréversibles (aux conséquences permanentes) ont lieu. Il n'existe pas de période critique générale lors de la différenciation sexuelle du cerveau, mais une succession d'étapes développementales propres à chaque système neuronique et qui sont arrangées en parallèle et en série. A priori, la période critique (début et fin) peut être déterminée génétiquement ou être secondaire à un événement déterminé épigénétiquement. Ainsi, les différentes compo- 
santes du développement d'un neurone (genèse, migration, poussée neuritique, élaboration des connexions et acquisition pharmacologique) peuvent obéir à des règles différentes ce qui augmente le potentiel d'expression fonctionnelle de la différenciation sexuelle (Figure 6 ).

5. Mutagenèse dirigée : récepteurs auX GONADOSTÉROÏDES ET AROMATASE [5, 7, 30, 31]

Des lignées de souris transgéniques ont été récemment obtenues en laboratoire portant des inactivations sur les gènes de $\mathrm{ER} \alpha, \mathrm{ER} \beta$, ER $\alpha \beta$, PR et AROM. Concernant la différenciation sexuelle, seule une étude neuroanatomique du cerveau des souris déficientes en $\mathrm{ER} \alpha(\alpha \mathrm{ERKO})$ est disponible à ce jour.

Les différentes lignées mutantes présentent toutes des déficits plus ou moins marqués de la fonction de reproduction. Les atteintes touchent l'appareil reproducteur, l'endocrinologie et/ou le comportement sexuels, chaque mutation permettant de préciser l'implication fonctionnelle prépondérante de tel ou tel récepteur, en particulier concernant la dichotomie ER/AR ou ER $\alpha / \mathrm{ER} \beta$.

Cependant, en dépit d'une somme considérable d'informations nouvelles apportées par l'étude de ces différents phénotypes, des relations causales sont en général difficiles à extraire concernant les rapports structure/fonction du cerveau, non seulement parce que ER $\alpha$, ER $\beta$, $A R$ et AROM coexistent dans de nombreux circuits neuroniques, mais aussi parce que les mutations touchent simultanément tous les étages de l'axe hypothalamo-hypophyso-gonadique.

De plus, l'inactivation d'un gène conduit à la suppression de son influence pendant tout le développement de l'individu, ce qui peut donner lieu à des phénomènes de compensation/ réorganisation ou encore se traduire par des dysfonctionnements secondaires, indirects et retardés.

Enfin, ces mutations sont induites chez la souris, une espèce chez laquelle relativement peu d'informations sont encore disponibles concernant la différenciation sexuelle du cerveau.
Surtout, il n'y a pas de modèle universel de la sexualisation du cerveau : par exemple, il n'existe pas de SDN histologiquement identifiable chez la souris.

Si l'on s'en tient à l'examen des conséquences comportementales des inactivations géniques, les études disponibles à l'heure actuelle confirment les résultats obtenus précédemment à l'aide des antagonistes des ER et AR et des inhibiteurs de l'AROM.

La seule étude disponible et réalisée chez la souris $\alpha$ ERKO rapporte l'absence de masculinisation d'une population neuronale dopaminergique de l'APO chez les mâles, population neuronique semblant normalement masculinisée chez les mâles porteurs d'un testicule féminisant. Ce résultat confirme le rôle masculinisateur de l'E2 précédemment démontré chez le rat par des expériences classiques de gonadectomie néonatale et traitement substitutif.

\section{LA DIFFERENCIATION SEXUEL- LE DU CERVEAU CHEZ L'HOMME}

La différenciation sexuelle fonctionnelle du cerveau des Primates présente des caractéristiques qui les éloignent des Rongeurs. En particulier, l'hypothalamus endocrinien ne serait pas irréversiblement sexualisé chez les Primates puisque d'une part, l'apparition d'une libération cyclique de gonadotrophines a été observée suite à une greffe ovarienne chez un Macaque mâle orchidectomisé à l'âge adulte, et puisque d'autre part, la rétroaction positive de l'E2 est observable chez des hommes orchidectomisés à l'âge adulte. Le versant fonctionnel endocrinien chez les Primates n'obéirait donc pas aux mêmes règles d'organisation que chez les Rongeurs. Quant au versant fonctionnel comportemental, certaines conclusions peuvent être avancées concernant l'espèce humaine.

La distribution dans le cerveau des neurones possédant ER et/ou AR est remarquablement similaire chez l'Homme et chez l'animal, ce qui suggère que certains des effets d'organisation et d'activation des stéroïdes sexuels sur le cerveau se retrouveront dans notre espèce $[10,11$, 27]. La difficulté d'expérimenter, d'une part, et d'autre part la rareté du matériel autopsique 
ปี
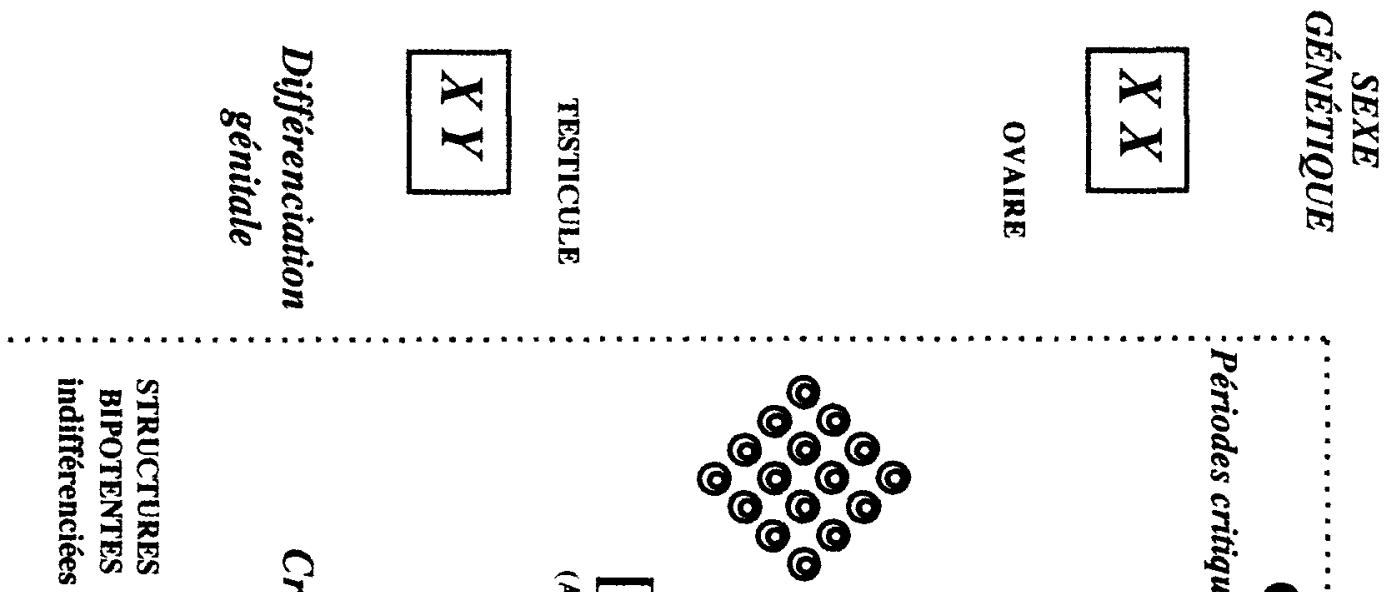

牙哭 क
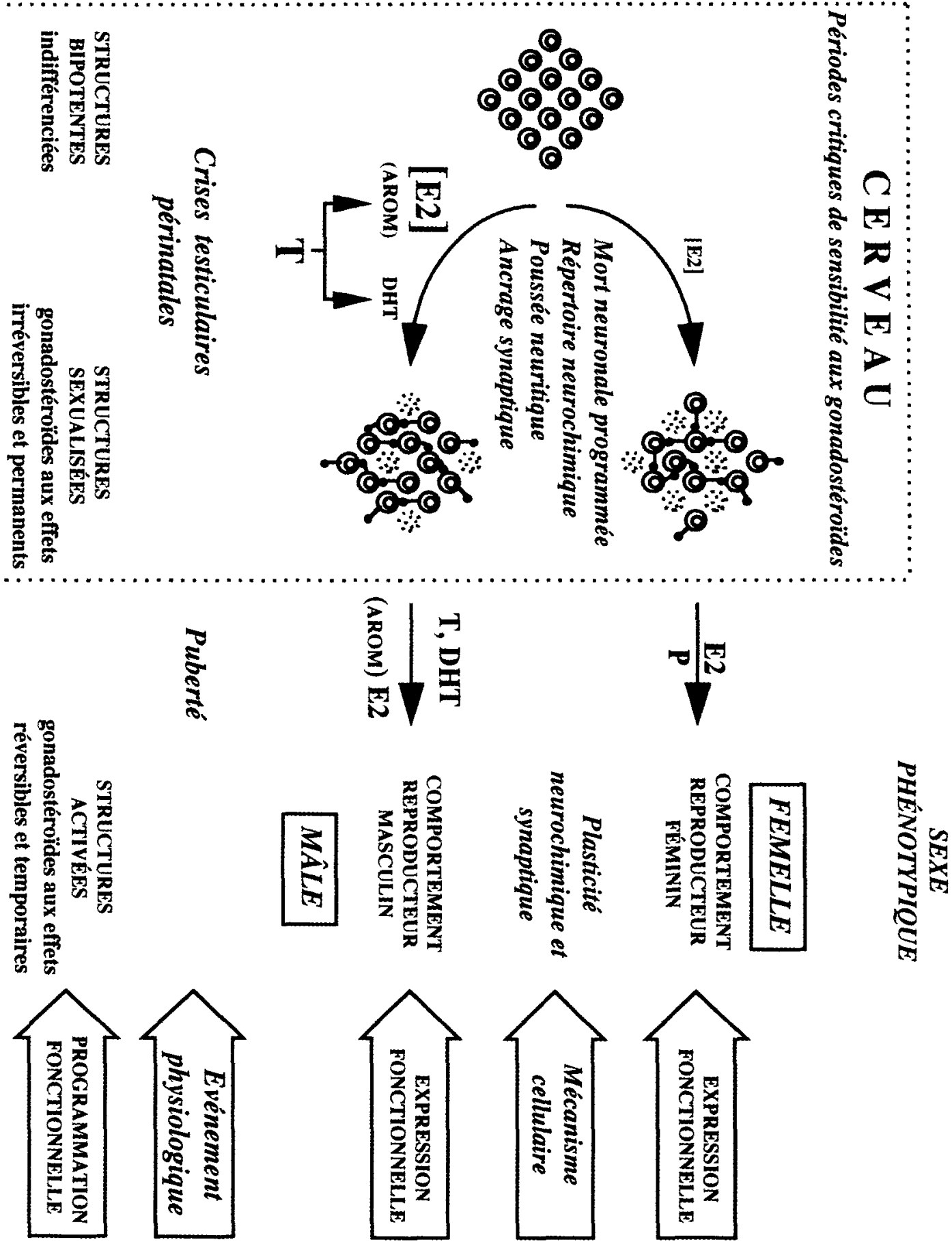
de qualité, expliquent une littérature encore relativement peu abondante ou controversée. Les études psychométriques, quant à elles, insistent souvent sur l'influence inhérente du contexte socioculturel dans la différenciation psychosexuelle. Les analyses bibliographiques rappellent souvent certains cas cliniques ou expériences qui permettent de formuler et de répondre à quatre questions fondamentales :

1) le système voméronasal est-il une réalité anatomofonctionnelle dans notre espèce?

2) Les stéroïdes sexuels sont-ils impliqués dans la différenciation sexuelle fonctionnelle du cerveau?

3) Peut-on trouver des dimorphismes sexuels dans l'organisation du cerveau ?

4) Peut-on rapprocher chez un même individu, atypie du dimorphisme anatomique et atypie du comportement?

\section{Organe voméronasal et communication PHÉROMONALE [12, 18, 26, 32].}

Après une assez longue période de controverse, plusieurs études rapportent l'identification histologique d'un organe voméronasal chez l'Homme. Si sa fonctionnalité n'est pas unanimement reconnue, plusieurs expériences démontrent l'existence d'une communication phéromonale sexualisée dans notre espèce. Les aisselles des hommes produisent l'androsta4,16-diène-3-one qui aurait des vertus apaisantes sur les sujets féminins, et les aisselles des femmes produiraient deux facteurs non encore identifiés qui provoqueraient la synchronisation interindividuelle du pic préovulatoire de gonadotrophines.

Nous pouvons retenir aujourd'hui qu'en dépit d'incertitudes, la littérature comporte assez d'éléments d'information permettant d'envisager l'existence et la fonctionnalité de l'organe voméronasal dans notre espèce, et donc celles du système voméronasal, c'est-à-dire celle du méta-réseau cérébral sexuellement différencié et bien décrit chez l'animal.

2. Différenciation PSychosexuelle : SYNDROMES CLINIQUES [3, 4, 16, 22, 23, 25, 34, $36,37,38]$.

Des mutations inactivatrices sur le gène de l'enzyme 21-hydroxylase résultent en une hypersécrétion de précurseurs androgéniques par la surrénale embryonnaire, ce qui conduit à une virilisation génitale plus ou moins prononcée des filles à la naissance. Ce syndrome d'hyperplasie surrénalienne congénitale (HSC) est rapidement diagnostiqué, les filles sont socialement reconnues comme telles, reçoivent une chirurgie correctrice et sont traitées pour leur insuffisance surrénalienne. Ces individus, qui se comportent souvent comme des garçons manqués durant leur enfance, montreront à l'âge adulte une propension significative à s'investir professionnellement plutôt que maternellement. Certaines études psychométriques leur accordent, une identité sexuelle féminine et une orientation hétérosexuelle, toutes deux bien marquées, mais une tendance significativement supérieure à la moyenne à l'élaboration de fantasmes bi- ou homosexuels. Ces observations suggèrent que l'empreinte androgénique périnatale a influencé la différenciation sexuelle fonctionnelle du cerveau. Un tel syndrome d'androgénisation des fœtus femelles se retrouve systématisé par l'évolution chez la Hyène Tachetée, espèce chez laquelle les femelles sont dominantes pour compenser la rudesse de la compétition alimentaire en savane africaine. Durant la gestation, les ovaires maternels produisent de fortes concentrations d'androstènedione converties en $\mathrm{T}$ par le placenta. Le fotus femelle est masculinisé, les adultes ont un clitoris péniforme érectile et un SDN partiellement masculinisé. Le comportement sexuel des femelles est hétérosexuel avec des tentatives de monte désorganisées, et elles démontrent une forte agressivité dès l'enfance.

La description de cas remarquables d'individus $\mathrm{XY}$ porteurs de testicules féminisants ( $\mathrm{Tfm}$ ) avec une insensibilité complète aux androgènes, un phénotype externe féminin, une identité sexuelle féminine et une orientation sexuelle univoque pour les hommes laisse à penser que, dans notre espèce, la masculinisation fonctionnelle définitive du cerveau est essentiellement réalisée par les AR.

Un rôle masculinisateur des ER durant l'organogenèse cérébrale est difficile à envisager à partir de la littérature clinique actuelle. Les individus $\mathrm{XX}$ dont les mères reçurent un trai- 
tement au diéthylstilbestrol durant leur grossesse présenteraient un profil psychosexuel comparable aux patients HSC. Certains auteurs suggèrent que la différenciation psychosexuelle pourrait reposer sur une empreinte double, cstrogénique prénatale (préparatoire ou facilitatrice) qui viendrait s'ajouter à l'empreinte androgénique prénatale et postnatale sur le cerveau des garçons.

Si les individus Tfm permettent d'envisager des effets d'organisation pour les androgènes sur le cerveau humain, les effets d'activation des androgènes sur le comportement sexuel sont connus, et des cas spectaculaires de masculinisation tardive à la puberté chez les garçons sont bien décrits pour certains syndromes de déficience (androgénique) de la $5 \alpha$-réductase 2 ou de la $17 \beta$-hydroxystéroïde déhydrogénase 3 .

À notre connaissance, il n'existe pas d'étude histologique concernant les cerveaux de ces différents phénotypes.

\section{Dimorphismes sexuels dans le cerveau HUMAIN.}

Il est bien établi qu'il existe chez l'Homme un dimorphisme sexuel dans certaines fonctions cognitives et dans la prévalence de certaines pathologies neurologiques ou psychiatriques, et des dimorphismes ont été découverts dans plusieurs régions du cerveau.

Certains noyaux de l'hypothalamus ont été décrits comme sexuellement dimorphes. Des différences dans la nomenclature anatomique, l'échantillonnage et les procédures morphométriques entre les études publiées ont toutefois prêté à controverses.

Le groupe de $\mathrm{D}$. Swaab à Amsterdam a très largement contribué à l'étude des dimorphismes sexuels dans l'hypothalamus humain où ces auteurs reconnaissent un SDN identifiable histologiquement. Ils ont estimé que $80 \%$ des neurones du SDN sont générés en période postnatale et qu'un dimorphisme sexuel $(M>F)$ ne devient observable qu'à partir de quatre ans pour se stabiliser à la puberté. Cette sexualisation tardive (début d'apoptose neuronale chez les filles) du SDN dans notre espèce n'exclut pas que son développement soit programmé précocement in utero, et permet aussi d'évoquer la possible influence de facteurs épigénétiques (traitements, environnement, chocs émotionnels, éducation) sur son développement ultérieur.

\section{Corrélats anatomiques à l'Homosexuali-} TÉ ET AU TRANSSEXUAIISME

Cette question est encore plus sensible que celle des dimorphismes sexuels, et la controverse est de règle. Les incertitudes sont relatives aux sujets examinés, souvent morts du sida, ainsi qu'aux procédures morphométriques utilisées. Sans prendre parti, citons deux exemples largement médiatisés.

Simon Le Vay publie en 1991 dans Science qu'un noyau de l'hypothalamus antérieur (INAH3) est deux fois plus volumineux chez les hommes hétérosexuels que chez les femmes et les hommes homosexuels, suggérant que l'orientation sexuelle pourrait avoir une base neurologique.

Le groupe de D. Swaab publie dans Nature en 1995 et dans The Journal of Clinical Endocrinology \& Metabolism en 2000 une différence d'organisation dans une structure voméronasale, le noyau du lit de la strie terminale (NLST). Le NLST est sexuellement dimorphe, il contient plus de neurones chez les hommes $(n=9)$ que chez les femmes $(n=9)$. Il n'est pas statistiquement différent chez les hommes hétéro- $(n=9)$ et homosexuels $(n=9)$ mais est féminisé chez les transsexuels XY $(n=6)$. De plus, un cas unique de transsexuel $\mathrm{XX}$ présente un noyau masculinisé. Cette étude suggère que l'identité sexuelle pourrait avoir une base neurologique.

Si l'APO où se trouve INAH3 et si le NLST sont des structures clairement impliquées dans le comportement reproducteur de tous les mammifères étudiés à ce jour, il est certainement illégitime de conclure qu'orientation sexuelle et identité sexuelle y sont respectivement localisées. Ces deux composantes de la fonction de reproduction sont les manifestations de l'activité de réseaux entiers et l'on attend des données supplémentaires à partir de ce même matériel autopsique concernant les régions connectées à INAH3 et au NLST. Toutefois, nous pouvons considérer qu'ont été jetées les bases d'une recherche des rapports 
structure-fonction pour le comportement sexuel que nous pouvons le mieux appréhender, c'est-à-dire celui de notre propre espèce. L'étude des cerveaux des transsexuels XY a consolidé notre connaissance des mécanismes de la différenciation du cerveau humain. Ces sujets sont semblables en ce qui concerne la taille du NLST mais dissemblables en ce qui concernait leur état endocrinien avant l'autopsie (orchidectomie, date et durée des traitements). Il existe donc une incohérence double entre le sexe phénotypique cérébral, d'une part, et d'autre part le sexe génétique et l'état endocrinien à l'âge adulte. Cette observation suggère que la taille du NLST ne dépend pas de l'action postnatale des gonadostéroïdes et rappelle les résultats des expériences de renversement du sexe phénotypique de l'APO chez les Rongeurs qui ont démontré l'action précoce, organisatrice, irréversible et permanente de la gonade sur le cerveau. La nature du dysfonctionnement périphérique ou central à l'origine de l'atypie d'organisation du NLST des transsexuels XY est inconnue.

Quelque différents ou semblables que puissent être les mécanismes développementaux et l'organisation des réseaux à compétence reproductive entre les Rongeurs et les Primates, il est clair que l'important développement du néocortex chez les Primates impose inévitablement de débattre des parts relatives de l'inné et de l'acquis. C'est justement toute la question de la différenciation sexuelle du cerveau et de ses conséquences sur la nature du traitement central des émotions et des rapports sociaux.

\section{CONCLUSION}

La biologie de la reproduction ne se comprend bien que dans une perspective évolutionniste. Chaque espèce adapte sa stratégie de reproduction en fonction de son milieu et de son histoire biologique. En cela, les dimorphismes sexuels de l'anatomie et la chimie du cerveau peuvent être conçus comme résultant de contraintes et nécessaires à l'émergence d'une différenciation fonctionnelle. Ce raisonnement permet de rapprocher structure et fonction dans des cas univoques. Ainsi, certains motoneurones de la moelle lombaire des
Mammifères subsistent chez le mâle pour assurer la motilité d'un organe absent chez la femelle. Ou encore dans le cerveau d'oiseaux chanteurs (les Canaris), les zones du contrôle du chant sont peu développées chez la femelle qui ne chante pas. Cependant, de tels exemples pour lesquels le rapport structure-fonction saute littéralement aux yeux sont des exceptions. Le cas du SDN est prototypique : en dépit d'une somme considérable d'efforts dans ce sens, les recherches sur la conséquence fonctionnelle de son dimorphisme n'ont abouti à aucune conclusion ferme. Si l'APO semble être essentielle pour l'expression du comportement sexuel masculin, ni la fonction particulièrement dévolue au SDN, ni la nécessité de neurones surnuméraires à cet endroit n'ont été éclaircies.

À vrai dire, la difficulté rencontrée lors de la recherche d'une signification physiologique ou comportementale à la différenciation sexuelle de telle ou telle région centrale provient de l'extraordinaire complexité et hétérogénéité locale du cerveau. Par exemple, il est aujourd'hui pratiquement impossible de détruire (ou cibler par un traitement) de façon strictement élective un circuit précis dans sa totalité. Très souvent, des phénomènes de compensation viennent obscurcir le résultat final. D'autre part, les techniques morphologiques qui permettent de mettre en évidence l'activation neuronale ont révélé la participation plurifonctionnelle des régions sexuellement différenciées qui peuvent être impliquées à la fois dans des réponses endocriniennes et comportementales, ou encore dans deux composantes distinctes d'un même comportement. À peine commençons-nous à entrevoir l'extraordinaire potentiel de plasticité fonctionnelle des réseaux neuroniques les plus simples.

L'étude de la différenciation sexuelle du cerveau a pourtant permis d'obtenir une vision concrète des problèmes posés à la recherche des rapports structure-fonction dans le système limbique. Cette discipline, à la frontière entre embryologie, endocrinologie et neurobiologie a aussi grandement élargi et enrichi le champ d'investigation des rôles des stéroïdes sexuels dans le métabolisme cérébral et ainsi fertilisé l'ensemble des neurosciences. 


\section{REMERCIEMENTS}

Je remercie le Pr. Gérard Tramu (Université de Bordeaux I) pour sa lecture critique du manuscrit et son constant soutien ainsi que l'INSERM, l'Université de Bordeaux 2-Victor Ségalen et la Fondation pour la Recherche Médicale.

\section{RÉFÉRENCES}

1. BAULIEU E.E. : Neurosteroids: a novel function of the brain. Psychoneuroendocrinology, 1998, 23 : 963-987.

2. BIMONTE A.H., FITCH R.H., DENENBERG V.H. : Neonatal estrogen blockade prevents normal callosal responsiveness to estradiol in adulthood. Dev. Brain Res., 2000, 122 : 149-155.

3. BYNE W., LASCO M.S., KEMETHER E. et al. : The interstitial nuclei of the human anterior hypothalamus: an investigation of sexual variation in volume and cell size, number and density. Brain Res., 2000, 856 : 254-258.

4. CARANI C., ROCHIRA V., FAUSTINI-FUSTINI M., BALESTRIERI A., GRANATA A.R.M. : Role of oestrogen in male sexual behavior: insights from the natural model of aromatase deficiency. Clin. Endocrinol., 1999, 51 : 517-524.

5. CHAPPELL P.E., SCHNEIDER J.S., KIM P., XU $M$. et al. : Absence of gonadotropin surges and gonadotropin-releasing hormone self-priming in ovariectomized (OVX), estrogen (E2)-treated, progesterone receptor knockout (PRKO) mice. Endocrinology, 1999, 140 : 3653-3658.

6. COOKE B., HEGSTROM C.D., VILLENEUVE L.S., BREEDLOVE S.M. : Sexual differentiation of the vertebrate brain: principles and mechanisms. Front. Neuroendocrinol., 1998, 19 : 323362.

7. COUSE J.F., KORACH K.S. : Estrogen receptor null mice: What have we learned and where will they lead us ? Endocr. Rev., 1999, 20 : 358417.

8. DAVIS E.C., POPPER P., GORSKI R.A. : The role of apoptosis in sexual differentiation of the rat sexually dimorphic nucleus of the preoptic area. Brain Res., 1996, 734 : 10-18.

9. DE VRIES G.J., MILLER M.A. : Anatomy and function of extrahypothalamic vasopressin systems in the brain. Prog. Brain Res., 1998, 119 : 320.

10. DONAHUE J.E., STOPA E.G., CHORSKY R.L. et al. : Cells containing immunoreactive estrogen receptor-a in the human basal forebrain. Brain Res., 2000, 856 : 142-151.

11. FERNANDEZ-GUASTI A., KRUIJVER F.P., FODOR M., SWAAB D.F. : Sex differences in the distribution of androgen receptors in the human hypothalamus. J. Comp. Neurol., 2000, 425 : 422-435.

12. GROSSER B.I., MONTI-BLOCH L., JENNINGSWHITE C., BERLINER D.L. : Behavioral and electrophysiological effects of androstadienone, a human pheromone. Psychoneuroendocrinology, 2000, 25 : 289-299

13. GUILLAMON A., SEGOVIA S. : Sex differences in the vomeronasal system. Brain. Res. Bull., 1997, 44 : 377-382.

14. HARRIS G.W. : Hormonal differentiation of the developing central nervous sytem with respect to patterns of endocrine function. Phil. Trans. Roy. Soc. Lond., 1970, 259 : 165-177.

15. HERBISON A.E. : Multimodal influence of estrogen upon gonadotropin-releasing hormone neurons. Endocr. Rev., 1998, 19 : 302-330.

16. HINES M. : Abnormal sexual development and psychosexual issues. Baillieres Clin. Endocrinol. Metab., 1998, 12 : 173-89.

17. HUTCHISON J.B., WOZNIAK A., BEYER C., KAROLCZAK M., HUTCHISON R.E. : Steroid metabolising enzymes in the determination of brain gender. J. Steroid Biochem. Mol. Biol., 1999, 69 : 85-96.

18. JAHNKE V., MERKER H.J. : Electron microscopic and functional aspects of the human vomeronasal organ. Am. J. Rhinol., 2000, 14 : 63-67.

19. JORDAN C.L. : Glia as mediators of steroid hormone action on the nervous system: An overview. J. Neurobiol., 1999, 40 : 434-445.

20. JOST A. : Les péripéties d'une recherche : l'étude de la différenciation sexuelle. La Vie des Sciences, 1991, 8 : 67-87.

21. KNOBIL E., HOTCHKISS J. : The menstrual cycle and its neuroendocrine control. In : Knobil E., Neill J.D., Ewing L.L., et al., eds. Physiology of Reproduction, 2nd Ed., vol. 2. New York, Raven Press, 1988 : 1971-1994.

22. KRUIJVER F.P.M., ZHOU J.-N., POOL C.W., HOFMAN M.A., GOOREN L.J.G., SWAAB D.F. : Male-to-female transsexuals have female neuron numbers in a limbic nucleus. J. Clin. Endocrinol. Metab., 2000, 85 : 2034-2041.

23. LE VAY S. Le Cerveau a-t-il un Sexe ? Nouvelle Bibliothèque Scientifique. Paris, Flammarion, 1994.

24. McEWEN B.S., ALVES S.E. : Estrogen actions in the central nervous system. Endocr. Rev., 1999, 20 : 279-307.

25. MEYER-BAHLBURG H.F.L., EHRHARDT A.A., ROSEN L.R. et al. : Prenatal estrogens and the development of homosexual orientation. Dev. Psychol., 1995, 31 : 12-21.

26. MONTI-BLOCH L., JENNINGS-WHITE C., BERLINER D.L. : The human vomeronasal system. A review. Ann. N. Y. Acad. Sci., 1998, 855: 373-389.

27. OSTERLUND M.K., GRANDIEN K., KELLER E., 
HURD Y.L. : The human brain has distinct regional expression patterns of estrogen receptor alpha mRNA isoforms derived from alternative promoters. J. Neurochem., 2000, 75 : 13901397.

28. SCHUMACHER M., COIRINI H., ROBERT F., GUENNOUN R., EL-ETR M. : Genomic and membrane actions of progesterone: implications for reproductive physiology and behavior. Behav. Brain Res., 1999, 105 : 37-52.

29. SEGOVIA S., GULLAMON A., DEL CERRO M.C. et al. : The development of brain sex differences: a multisignaling process. Behav. Brain Res., 1999, 105 : 69-80.

30. SIMERLY R.B., ZEE M.C., PENDLETON J.W., LUBAHN D.B., KORACH K.S. : Estrogen receptor-dependent sexual differentiation of dopaminergic neurons in the preoptic region of the mouse. Proc. Natl. Acad. USA, 1997, 94 : 1407714082.

31. SIMPSON E.R. : Genetic mutations resulting in loss of aromatase activity in humans and mice. J. Soc. Gynecol. Investig., 2000,7 : S18-21.

32. STERN K., McCLINTOCK M.K. : Regulation of ovulation by human pheromones. Nature, 1998, 392 : 177-179.

33. STOMATI M., GENAZZANI A.D., PETRAGLIA F., GENAZZANI A.R. : Contraception as prevention and therapy: sex steroids and the brain. Eur. J. Contracept. Reprod. Health Care, 1998, 3 : 21-28.

34. SWAAB D.F., HOFMAN M.A. : Sexual differentiation of the human hypothalamus in relation to gender and sexual orientation. Trends Neurosci., 1995, 18 : 264-270.

35. TORAN-ALLERAND C.D., SINGH M., SETALO G.Jr. : Novel mechanisms of estrogen action in the brain: new players in an old story. Front. Neuroendocrinol., 1999, 20 : 97-121.

36. WHITE P.C., SPEISER P.W. Congenital adrenal hyperplasia due to 21-hydroxylase deficiency. Endocr. Rev., 2000, 21 : 245-291.

37. WILSON J.D. : The role of androgens in male gender role behavior. Endocr. Rev., 1999, 20 : 726-737.

38. WISNIEWSKI A.B., MIFEON C.J., MEYERBAHLBURG H.F.L. et al. : Complete androgen insensitivity syndrome: Long-term medical, surgical, and psychosexual outcome. J. Clin. Endocrinol. Metab., 2000, 85 : 2664-2669.

\section{Sexual differentiation of the brain by gonadal steroids. A minireview.}

\section{P. CIOFI}

This review presents the basic concepts regarding sexual differentiation of the brain derived from experimental studies in laboratory animals. Although sexual differentiation of the brain sex is genetically and epigenetically determined, only epigenetic gonadal steroidal factors are discussed here. Sexual differentiation of the anatomy and chemistry of the central nervous system is essential to ensure functional differentiation, to meet the body's differing needs imposed by the presence of an ovary or a testis. During embryonic development of the brain, the gonad therefore imposes its own sex on a number of brain neural circuits that constitute the broad network controlling reproduction, the vomeronasal system, comprising the upstream pheromonesensing vomeronasal organ whose activation triggers both endocrine and behavioral downstream adaptive responses following hypothalamic integration. In many ways, sexual differentiation of the brain resembles sexual differentiation of the genital tract: the testis diverts a bipotential structure away from a feminine differentiation program. Masculinisation of the brain takes place early in development during critical periods (perinatal in many species) of sensitivity of the nervous tissue to the testicular imprint. Testosterone acts to permanently and irreversibly organise virtually every possible feature of nervous tissue: numbers and shapes of glial cells or neurons, axons, dendrites, synapses, cell metabolism, repertoire of neurotransmitters and receptors. The precise mechanisms of sexual differentiation vary in different brain regions. Although testosterone often appears to act alone after aromatisation into estradiol in neurons, masculinisation may also be determined by purely androgenic or even mixed, androgenic-estrogenic mechanisms. These steroidal imprints ultimately control neuronal apoptosis, neuritic extension and specialisation and chemical phenotypes. Because of its spectacular impact, the influence of the testis on brain sexual differentiation has received a great deal of attention. However, experimentally-induced absen- 
ce of testicular imprint in the male brain does not systematically lead to the inherent development of feminine features, as growing evidence suggests the necessity of the perinatal ovary to ensure definitive expression of certain female features. For example, a female organisation is not the default program of brain development as observed in the genital tract. Sexual dimorphisms of anatomy and chemistry are also clearly observed in the human brain but, as in animals, structure/f unction relationships are more difficult to define, essentially because of the marked complexity and local heterogeneity of brain tissue. Nevertheless, post-mortem histological studies of brains from homosexual and transsexual individuals with sufficient clinical records suggest that specific elements of sexual behaviour such as sexual orientation or sexual identity have a biological basis. Therefore, in humans as in animals, sexual behaviour, i.e. certain aspects of social behaviour, may partly depend on innate features.

Key-words: brain sexual differentiation, sexual dimorphisms, hypothalamus, estradiol, testosterone, gonadoliberin. 